
MOTHERS WITH SYSTEMIC AUTOIMMUNE DISEASES: A MULTICENTRE ITALIAN SURVEY FROM 24 RHEUMATOLOGY CENTRES

M.G. Lazzaroni ${ }^{1}$, C. Nalli ${ }^{1}$, L. Andreolit ${ }^{1}$, C. Carini ${ }^{1}$, E. Bartoloni-Bocci ${ }^{2}$, R. Gerli ${ }^{2}$, C. B. Chighizola ${ }^{3}$, M. Gerosa ${ }^{3}$, P.L. Meroni ${ }^{3}$, L. Sinigagliaa ${ }^{3}$, P. Conigliaro ${ }^{4}$,

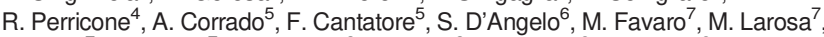
A. Doria ${ }^{7}$, A. Ruffatti ${ }^{7}$, E. Generali ${ }^{3}$, C. Selmi ${ }^{3}$, M. Meroni ${ }^{8}$, M. Cutolo $^{8}$, M. Padovan ${ }^{9}$, M. Govoni ${ }^{9}$, G. Pazzola ${ }^{10}$, C. Salvarani ${ }^{10}$, S. Peccatori ${ }^{11}$ G. Paolazzi ${ }^{11}$, I. Prevete ${ }^{4}$, G.D. Sebastiani ${ }^{4}$, G. Minisola ${ }^{4}$, A. Brucato ${ }^{12}$, V. Ramoni ${ }^{13}$, R. Caporali ${ }^{13}$, C. Montecucco ${ }^{13}$, C. Tani ${ }^{14}$, V. Signorini ${ }^{14}$, M. Mosca ${ }^{14}$, M. Trevisani ${ }^{15}$, N. Malavolta ${ }^{15}$, M. Vadacca $^{4}$, A. Afeltra ${ }^{4}$, E. Vivaldelli $i^{16}$, A. Maier ${ }^{16}$, E. Visalli $^{17}$, R. Foti ${ }^{17}$, L. Zuliani $^{18}$, A. Gabrielli ${ }^{18}$, C. Campochiaro ${ }^{3}$, E. Baldissera ${ }^{3}$, M.G. Sabbadini ${ }^{3}$, N. Romeo ${ }^{19}$, A. Tincani ${ }^{1}{ }^{1}$-, Brescia; ${ }^{2}$, Perugia; ${ }^{3}$-, Milano; ${ }^{4}$-, Roma; ${ }^{5}{ }_{-}$, Foggia; $^{6}{ }_{-}$, Potenza; ${ }^{7}$, Padova; ${ }^{8}{ }_{-}$,

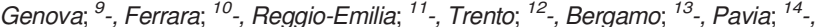
Pisa; ${ }^{15}$-, Bologna; ${ }^{16}{ }_{-}$, Bolzano; ${ }^{17}$-, Catania; ${ }^{18}$-, Ancona; ${ }^{19}{ }_{-}$, Cuneo, Italy

Background: Rheumatic Diseases (RD) frequently affect women during reproductive age, therefore counselling on family planning is crucial for their quality of life. Children's outcome is a major topic, but no large studies are available.

Objectives: To assess the long-term health conditions of children born to women with RD in a large multicentre cohort.

Methods: 24 Rheumatology Centres distributed the questionnaire 65 multiplechoice and 12 open-answer questions) to consecutive patients (18-55 years) in September 2015. Data were analysed dividing children upon maternal diagnosis: Chronic Arthritides (CA) and Connective Tissue Diseases (CTD).

Results: Data were collected for 320 children (166 males, 52\%) born to 184 mothers (63 CA and $121 \mathrm{CTD}$ ). At the time of interview, children had a mean age of $17.1 \pm 9.6$ years. Preterm delivery $(<37 \mathrm{w})$ was observed in 72 cases $(22.5 \%)$, including $13(4 \%)<34 \mathrm{w}$. Data on autoimmune/inflammatory disease (AIID) and/or neurodevelopmental disorders (ND)/learning disabilities (LD) is reported in table 1. 12 children (3.7\%) had a diagnosis of AlID, mostly coeliac disease $(8 / 12,67 \%)$ and 11 children (3.4\%) of a ND and/or LD by a Paediatric Neuropsychiatrist. To rule out the possible effects of in utero exposure to maternal autoantibodies and/ or anti-rheumatic drugs in the pathogenesis of ND, these data were retrieved for 280 children $(88 \%)$ and a comparison was performed between 11 affected and 269 not-affected children (table 2).

Abstract FRI0373 - Table 1. DM: diabetes mellitus; LD: learning disorder; ADHD: attention deficit hyperactivity disorder

\begin{tabular}{|c|c|c|c|}
\hline $\begin{array}{l}\text { NEURODEVELOPMENTAL/ } \\
\text { LEARNING DISORDERS }\end{array}$ & $\begin{array}{c}\text { Present } \\
(n=11)\end{array}$ & $\begin{array}{c}\text { Absent } \\
(n=269)\end{array}$ & $\begin{array}{c}\mathrm{p}- \\
\text { value }\end{array}$ \\
\hline Male & $7(64 \%)$ & $140(55 \%)$ & 0.55 \\
\hline $\begin{array}{l}\text { Preterm birth } \\
<37 \text { weeks } \\
<34 \text { weeks }\end{array}$ & $\begin{array}{l}3(27 \%) \\
1(9 \%)\end{array}$ & $\begin{array}{l}56(22 \%) \\
10(3.7 \%)\end{array}$ & $\begin{array}{l}0.44 \\
0.36\end{array}$ \\
\hline $\begin{array}{l}\text { Maternal diagnosis } \\
\text { Chronic Arthritides } \\
\text { Connective Tissue Diseases }\end{array}$ & $\begin{array}{l}2(18 \%) \\
9(82 \%)\end{array}$ & $\begin{array}{l}95(37 \%) \\
174(63 \%)\end{array}$ & 0.34 \\
\hline Birth weight (mean $\mathrm{Kg}$ ) & 2.958 & 3.218 & 0.09 \\
\hline $\begin{array}{l}\text { In utero exposure to } \\
\text { anti-rheumatic drugs }\end{array}$ & $5(45 \%)$ & $63(24 \%)$ & 0.14 \\
\hline Prednisone & $5(45 \%)$ & $46(17 \%)$ & 0.22 \\
\hline Hydroxychloroquine & $0(0 \%)$ & $23(9 \%)$ & 0.61 \\
\hline Azathioprine & $1(9 \%)$ & $1(0,4 \%)$ & 0.08 \\
\hline $\begin{array}{l}\text { In utero exposure to } \\
\text { maternal auto-antibodies }\end{array}$ & $4(36 \%)$ & $54(20 \%)$ & 0.99 \\
\hline Anti-nuclear & $4(36 \%)$ & $46(17 \%)$ & 0.64 \\
\hline Anti-dsDNA & $3(27 \%)$ & $14(5 \%)$ & 0.06 \\
\hline Anti-Ro/SSA & $2(18 \%)$ & $24(9 \%)$ & 0.99 \\
\hline Anti-cardiolipin & $2(18 \%)$ & $15(6 \%)$ & 0.28 \\
\hline Anti-beta2gpl & $0(0 \%)$ & $12(4 \%)$ & 0.99 \\
\hline Lupus Anticoagulant & $0(0 \%)$ & $8(3 \%)$ & 0.99 \\
\hline
\end{tabular}

Abstract FRI0373 - Table 2

\begin{tabular}{|c|c|c|c|}
\hline $\begin{array}{l}\text { NEURODEVELOPMENTAL/ } \\
\text { LEARNING DISORDERS }\end{array}$ & $\begin{array}{l}\text { Present } \\
(n=11)\end{array}$ & $\begin{array}{l}\text { Absent } \\
(n=269)\end{array}$ & $\begin{array}{c}\text { p- } \\
\text { value }\end{array}$ \\
\hline Male & $7(64 \%)$ & $140(55 \%)$ & 0.55 \\
\hline $\begin{array}{l}\text { Preterm birth } \\
<37 \text { weeks } \\
<34 \text { weeks }\end{array}$ & $\begin{array}{l}3(27 \%) \\
1(9 \%)\end{array}$ & $\begin{array}{l}56(22 \%) \\
10(3.7 \%)\end{array}$ & $\begin{array}{l}0.44 \\
0.36\end{array}$ \\
\hline $\begin{array}{l}\text { Maternal diagnosis } \\
\text { Chronic Arthritides } \\
\text { Connective Tissue Diseases }\end{array}$ & $\begin{array}{l}2(18 \%) \\
9(82 \%)\end{array}$ & $\begin{array}{l}95(37 \%) \\
174(63 \%)\end{array}$ & 0.34 \\
\hline Birth weight (mean Kg) & 2.958 & 3.218 & 0.09 \\
\hline $\begin{array}{l}\text { In utero exposure to } \\
\text { anti-rheumatic drugs } \\
\text { Prednisone } \\
\text { Hydroxychloroquine } \\
\text { Azathioprine }\end{array}$ & $\begin{array}{l}5(45 \%) \\
0(0 \%) \\
1(9 \%)\end{array}$ & $\begin{array}{c}46(17 \%) \\
23(9 \%) \\
1(0,4 \%)\end{array}$ & $\begin{array}{l}0.22 \\
0.61 \\
0.08\end{array}$ \\
\hline $\begin{array}{l}\text { In utero exposure to } \\
\text { maternal auto-antibodies }\end{array}$ & $4(36 \%)$ & $54(20 \%)$ & 0.99 \\
\hline Anti-nuclear & $4(36 \%)$ & $46(17 \%)$ & 0.64 \\
\hline Anti-dsDNA & $3(27 \%)$ & $14(5 \%)$ & 0.06 \\
\hline Anti-Ro/SSA & $2(18 \%)$ & $24(9 \%)$ & 0.99 \\
\hline Anti-cardiolipin & $2(18 \%)$ & $15(6 \%)$ & 0.28 \\
\hline Anti-beta2gpl & $0(0 \%)$ & $12(4 \%)$ & 0.99 \\
\hline Lupus Anticoagulant & $0(0 \%)$ & $8(3 \%)$ & 0.99 \\
\hline
\end{tabular}

Conclusions: In this long-term follow-up of children born to mothers with RD in this large, multicenter study of randomly interviewed women each AIID did not display a significantly increased frequency as compared to the literature; only coeliac showed a mild increased frequency. Children with LD had a tendency to cluster in the group of mothers with CTD, especially after maternal diagnosis $(4 / 63,6.3 \%)$, with a higher frequency as compared to general paediatric population. No significant relationships between ND/LD and prematurity, intrauterine drug exposure or maternal autoantibodies were identified.

Acknowledgements: Statistical analysis supported by an unrestricted grant by UCB Pharma. The authors wish to thank Patients Associations and Participants to the survey.

Disclosure of Interest: None declared

DOI: 10.1136/annrheumdis-2018-eular.3866

\section{FRI0374 MILDER CLINICAL PRESENTATION OF LUPUS NEPHRITIS AND IMPROVED RENAL SURVIVAL DURING THE LAST 50 YEARS: A MULTICENTRIC STUDY}

M. Gatto ${ }^{1}$, G. Moroni ${ }^{2}$, F. Raffiotta ${ }^{2}$, P.G. Vercelloni ${ }^{3}$, S. Quaglini ${ }^{4}$, L. Sacchi ${ }^{4}$ D. Gainfreda ${ }^{5}$, A. Vaglio ${ }^{5}$, R.A. Sinico ${ }^{3}$, A. Doria $1 .{ }^{1}$ Rheumatology, University of Padova, Padova; ${ }^{2}$ Nephrology unit, Fondazione IRCCS Ca Granda Ospedale Maggioew Policlinico; ${ }^{3}$ Nephrology unit, University Milano Bicocca, Milano; ${ }^{4}$ Electrical, Computer and Biomedical Engineering, University of Pavia, Pavia; ${ }^{5}$ Nephrology unit, University Hospital Parma, Parma, Italy

Background: Lupus nephritis (LN) presentation changed over time following earlier diagnosis and treatment.

Objectives: To evaluate changes in LN clinical and histological presentation in the last 5 decades.

Methods: This is a retrospective multicentric study on prospectively collected data in four Italian hospital centres. Patients diagnosed between 1970 and 2016 were recruited provided they had a biopsy-proven LN that was retrospectively reclassified according to the ISN/RPS classification criteria. Follow-up was subdivided into three periods $(P)$ based on the year of LN diagnosis: P1:1970-1895; P2:1986-2000; P3:2001-2016. Predictors of patient and renal survival were investigated by univariate and multivariate analysis; survival curves were compared by log-rank test. Clinical pictures at presentation included isolated urinary abnormalities, nephrotic syndrome, nephritic syndrome, rapidly progressive renal failure. Outcome at last observation was defined as complete renal remission or 